\title{
Ikaros inhibits proliferation and, through upregulation of Slug, increases metastatic ability of ovarian serous adenocarcinoma cells
}

\author{
LI-CAI HE ${ }^{1 *}$, FENG-HOU GAO ${ }^{2 *}$, HAN-ZHANG XU ${ }^{1}$, SHAN ZHAO $^{1}$, \\ CHUN-MIN MA ${ }^{1}$, JUNE LI $^{3}$, SHU ZHANG ${ }^{4}$ and YING-LI WU ${ }^{1}$ \\ ${ }^{1}$ Department of Pathophysiology, Chemical Biology Division of Shanghai Universities E-Institutes, \\ Key Laboratory of Cell Differentiation and Apoptosis of National Ministry of Education, Rui-Jin Hospital, \\ Shanghai Jiao Tong University School of Medicine, Shanghai 200025; ${ }^{2}$ Number 3 People's Hospital, \\ Affiliated to Shanghai Jiao-Tong University School of Medicine (SJTU-SM), Shanghai 201900, P.R. China; \\ ${ }^{3}$ Department of Genetics, The University of Texas M.D. Anderson Cancer Center, Houston, TX 77030, USA; \\ ${ }^{4}$ Department of Obstetrics and Gynecology, Renji Hospital Affiliated to Shanghai Jiao Tong University, \\ School of Medicine (SJTU-SM), Shanghai 200125, P.R. China
}

Received May 16, 2012; Accepted July 13, 2012

DOI: 10.3892/or.2012.1946

\begin{abstract}
The transcription factor Ikaros was originally found to function as a key regulator of lymphocyte differentiation. In this study, we provide the first evidence that Ikaros is expressed at higher levels in ovarian cancer tissues compared with normal ovarian tissues and is significantly associated with high FIGO stage and low differentiation state in ovarian serous adenocarcinoma. To this end, we transfected IK1 (full length of Ikaros) into the SKOV3 ovarian cancer cell line and examined cell biological behaviors including proliferation, migration and invasion. We found that overexpression of IK1 inhibited cell proliferation by inducing $\mathrm{G}_{1}$ arrest, accompanied by the upregulation of P27 and P21 and downregulation of cyclin D1 and D2. On the other hand, IK1 increased the migration and invasion of ovarian cancer cells, as assessed by scratch-wound assay, transwell migration assay, and invasion assay. Overexpression of IK1 significantly increased Slug but not Snail1 expression at both mRNA and protein levels. It also downregulated and upregulated E-cadherin and MMP-2, two target genes of Slug involved in migration, respectively. Furthermore, knocking
\end{abstract}

Correspondence to: Dr Ying-Li Wu, Department of Pathophysiology, Key Laboratory of Cell Differentiation and Apoptosis of National Ministry of Education, Shanghai Jiao-Tong University School of Medicine (SJTU-SM), Shanghai 200025, P.R. China

E-mail: wuyingli@shsmu.edu.cn

Dr Shu Zhang, Department of Obstetrics and Gynecology, Renji Hospital Affiliated to Shanghai Jiao Tong University School of Medicine (SJTU-SM), Shanghai 200125, P.R. China

E-mail: drzhangshu@hotmail.com

*Contributed equally

Key words: Ikaros, metastasis, ovarian cancer, proliferation, Slug down Slug abrogated IK1-mediated increase in migration and invasion. These data suggest that Slug plays an important role in IK1-induced migration and invasion. In conclusion, we show for the first time that IK1 plays a dual role in the proliferation, migration and invasion of ovarian cancer cells, providing new insights into their metastasis.

\section{Introduction}

Ovarian cancer is one of the most common gynecological malignancies and the leading cause of death from gynecological cancers in women (1). Despite considerable efforts to improve early detection and the advances in chemotherapy, metastasis remains a major challenge in the clinical management of ovarian cancer. Approximately $70 \%$ of patients present with tumors that have spread beyond the ovaries (2). However, the mechanism through which a primary ovarian cancer cell develops into a metastatic phenotype is not well understood.

Ikaros, a member of a family of zinc finger transcription factors, is encoded by the IKZF1 gene (also known as ZNFN1A1) that comprises 8 exons. Alternative splicing of exons 3-6 can generate multiple Ikaros isoforms $(3,4)$. Ikaros was originally found to function as a critical regulator of lymphocyte differentiation. Subsequent studies reported that Ikaros also plays a role in hematopoietic stem cells and some myeloid cells (5-8). Moreover, Ikaros has also been shown to be expressed in mouse pituitary tissues, where it regulates the expression of adrenocorticotropic hormone and the adrenocortical hormone output (9), and several other tissues including liver, lung, prostate, brain, heart, placenta and intestine, in which the role of Ikaros is largely unknown. A recent report showed that the expression of Ikaros was correlated with the prognosis of several kinds of cancers including breast, lung, ovarian and skin cancers (10), suggesting a possible role of Ikaros in solid tumors. However, the expression and functional role of Ikaros in ovarian cancer have not been well studied. 
In this study, through immunohistochemical analysis, we found that Ikaros is expressed at higher levels in human ovarian cancer tissues than normal ovarian tissues, and Ikaros expression level is correlated with the different stages of ovarian serous adenocarcinoma cancer. Furthermore, we demonstrated that Ikaros may perform a dual role in ovarian cancer cells, that is, inhibiting cell proliferation and enhancing cell migration and invasion. We show that Slug (also known as Snail 2), an epithelial-mesenchymal transition associated protein, plays an important role in Ikaros-induced migration and invasion in ovarian cancer cells.

\section{Materials and methods}

Cell cultures. Human epithelial ovarian cancer cell line SKOV3 was obtained from ATCC (Manassas, VA). SKOV3 and HEK293T cell lines were cultured in RPMI-1640 medium (Sigma-Aldrich, St. Louis, MO) or Dulbecco's modified Eagle's medium (DMEM, Sigma-Aldrich) supplemented with $10 \%$ fetal bovine serum (FBS, Gibco BRL, Gaithersburg, MD). All cell lines were incubated in a $5 \% \mathrm{CO}_{2} / 95 \%$ air humidified atmosphere at $37^{\circ} \mathrm{C}$.

Retrovirus production and transduction of target cells. The pMSCV-puro-Flag-IK1 plasmid was obtained as reported previously (11). To produce virus, pGag-pol and pVSVG (Clontech, Palo Alto, CA) were co-transfected with pMSCV-puro-Ikaros or vehicle plasmid into 293T using FuGENE6 (Roche Applied Science, Basel, Switzerland) according to the manufacturer's instructions. Retrovirus-containing supernatant was harvested $48 \mathrm{~h}$ after transfection. The day before retrovirus infection, $3 \times 10^{5}$ cells were seeded in $2 \mathrm{ml}$ growth medium. On the next day, the growth medium was aspirated from the plate, $0.5 \mathrm{ml}$ growth medium was added, and $2 \mathrm{ml}$ retrovirus-containing supernatant was mixed with polybrene (Sigma, St. Louis, MO) to a final concentration of $2 \mu \mathrm{g} / \mathrm{ml}$. Forty-eight hours later, $1 \mu \mathrm{g} /$ $\mathrm{ml}$ puromycin (Sigma) was added to the medium. Positive polyclone population was identified based on Flag-IK1 expression.

Quantitative real-time polymerase chain reaction. Total cellular RNA was extracted by TRIzol kit (Invitrogen), followed by treatment with RNase-free DNase (Promega, Madison, WI). Complementary DNA was synthesized according to the manufacturer's instructions (Promega). Fluorescence real-time quantitative RT-PCR was performed with the double-stranded DNA dye SYBR Green PCR Core Reagents (PE Biosystems, Warrington, UK) using the ABI PRISM 7900 system (PerkinElmer, Torrance, CA). The specific primers used are shown in Table I. Real-time quantitative RT-PCR was performed and data were analyzed according to a previous report (12).

Tissue microarrays and immunohistochemistry. An ovarian cancer tissue array (US Biomax Inc., Rockville, MD) containing ovarian tumors from 87 patients and 10 cases of normal tissues were used for this study. Deparaffinization and antigen retrieval were accomplished by using Trilogy solution (Cell Marque, Rocklin, CA) and heating/pressure supplied by a conventional pressure cooker. Endogenous peroxidase activity was inhibited by using $0.3 \%$ hydrogen peroxide. Nonspecific interactions were blocked by using normal goat serum. Ikaros antibody (ab26083,
Abcam) was diluted (1:100) in PBS and incubated at $4^{\circ} \mathrm{C}$ overnight. Bound antibody was detected by using biotin-linked anti-rabbit secondary antibody and streptavidin-conjugated HRP enzyme in conjunction with DAB chromagen. Tissue was counterstained with hematoxylin. The immunoreactivity was defined by discrete brownish chromogen deposit in the cells. To quantify Ikaros expression, we further analyzed the cases with Ikaros expression by IPP (Image-Pro Plus, version 5.0, Media Cybernetics, Silver Spring, MD) using a method introduced by Xavier et al (13).

Cell proliferation assay. Cell proliferation was determined by Cell Counting kit- 8 assay (Dojindo, Japan), a sensitive nonradioactive colorimetric assay for determining cell growth, according to the manufacturer's instructions.

Cell cycle analysis. Briefly, Cells were fixed in $75 \%$ ethanol, resuspended in staining solution containing $50 \mu \mathrm{g} / \mathrm{ml}$ propidium iodide (PI) and $100 \mu \mathrm{g} / \mathrm{ml}$ RNase A, and incubated at $37^{\circ} \mathrm{C}$ for $30 \mathrm{~min}$. DNA content was analyzed by flow cytometry on a FACScan (Becton-Dickinson).

Scratch-wound assay. Cells were seeded to form a monolayer on 6-well plate surface and then a scratch wound was performed by dragging a sterile pipette tip across the layer. Detached cells were washed away with cell culture medium. An image was captured immediately by Olympus BX51 fluorescence microscope and the wound distance was calculated as a basic width. After $24 \mathrm{~h}$, cells were washed 3 times by PBS and another image was taken and the width of the wound distance was calculated. The wound closure (\%) was determined as the width migrated after $24 \mathrm{~h}$ relative to the basic width.

Transwell migration and invasion assay. Cell migration and invasion were gauged using a transwell migration assay and a matrigel invasion assay. Cell migration was examined using transwell chamber assay according to the protocol of the manufacturer (Costar). Briefly, 2.0x $10^{5}$ cells in RPMI-1640 plus $1 \%$ FBS in $200 \mu 1$ of RPMI-1640 were placed on each $8.0-\mu \mathrm{m}$ pore size upper chamber. RPMI-1640 plus $10 \%$ FBS was placed in the bottom wells as chemoattractant. The invasion assay used a BD Biocoatt Matrigelt Invasion Chamber (BD Biosciences, Bedford, MA, USA) with an 8.0- $\mu$ m pore size polyethylene terephthalate (PET) membrane coated with matrigel. The inserts were rehydrated by adding $0.5 \mathrm{ml}$ of warm culture medium at $37^{\circ} \mathrm{C}$ for $2 \mathrm{~h}$. The cells were seeded $\left(2.0 \times 10^{5}\right.$ cells in $0.5 \mathrm{ml}$ of serum-free medium) in the upper chamber and cultured as described in the method for the migration assay. After $24 \mathrm{~h}$, the nonmigrated or noninvaded cells on top of the membrane were gently removed with a cotton swab. Cells that had migrated or invaded were stained with $0.1 \%$ crystal violet (Sigma) and were counted and photographed under a microscope at a magnification of 400 in five to six randomly selected areas.

Western blot analysis. Western blotting was performed as previously described (11). Antibodies for western blotting are anti-Slug (9585), anti-Snail1 (3879), anti- $\beta$-catenin (9582), antiP21 (2947), anti-cyclin D1 (2922) from Cell Signaling, anti-P27 (Santa Cruz, SC-528), anti-flag (F1804, Sigma), anti-Ikaros (ab26083, Abcam), anti- $\beta$-actin (CP01, Calbiochem), and anti- 
Table I. Primers for real-time PCR.

\begin{tabular}{lll}
\hline Gene name & \multicolumn{1}{c}{ Forward primers } & \multicolumn{1}{c}{ Reverse primers } \\
\hline Snail1 & 5'-TGCCCTCAAGATGCACATCCGA-3' & 5'-GGGACAGGAGAAGGGCTTCTC-3' \\
Slug & 5'-ATCTGCGGCAAGGCGTTTTCCA-3' & 5'-GAGCCCTCAGATTTGACCTGTC-3' \\
$\beta$-catenin & 5'-CACAAGCAGAGTGCTGAAGGTG-3' & 5'-GATTCCTGAGAGTCCAAAGACAG-3' \\
E-cadherin & 5'-GCCTCCTGAAAAGAGAGTGGAAG-3' & 5'-TGGCAGTGTCTCTCCAAATCCG-3' \\
N-cadherin & 5'-CCTCCAGAGTTTACTGCCATGAC-3' & 5'-GTAGGATCTCCGCCACTGATTC-3' \\
MMP-2 & 5'-AGCGAGTGGATGCCGCCTTTAA-3' & 5'-CATTCCAGGCATCTGCGATGAG-3' \\
MMP-9 & 5'-GCCACTACTGTGCCTTTGAGTC-3' & 5'-CCCTCAGAGAATCGCCAGTACT-3' \\
GAPDH & 5'-CCACTCCTCCACCTTTGAC-3' & 5'-ACCCTGTTGCTGTAGCCA-3' \\
\hline
\end{tabular}

A
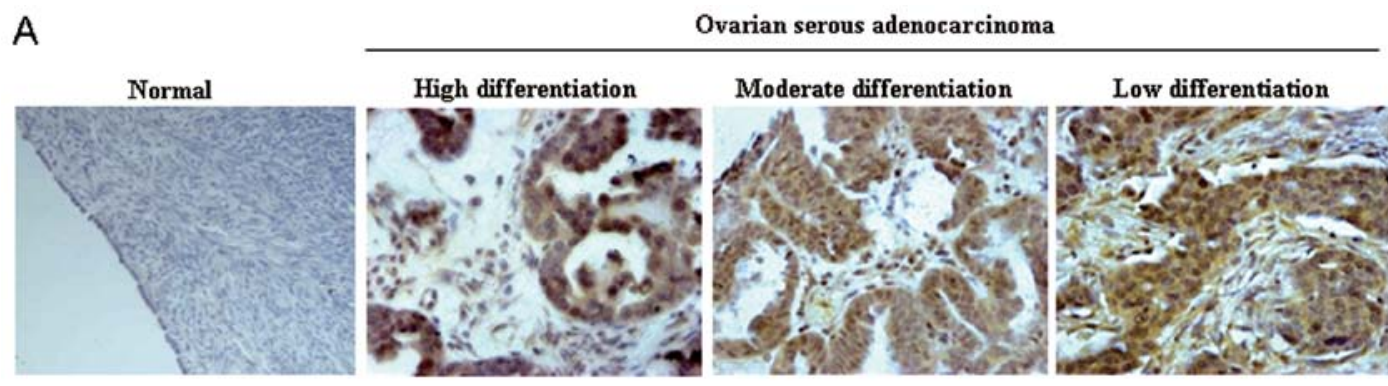

B

Ovarian serous adenocarcinoma

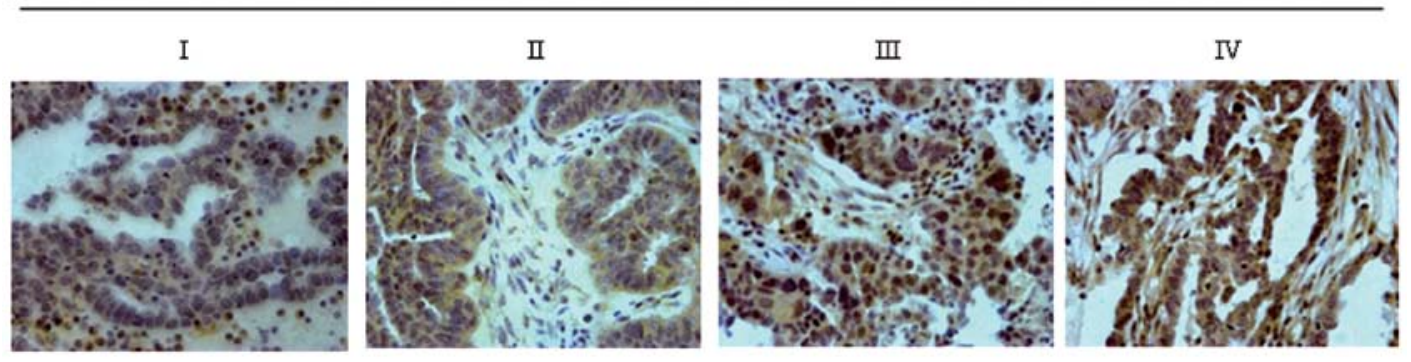

Figure 1. Ikaros is expressed at high level in human ovarian OSA tissues. Ikaros immunohistochemical expression was determined in normal and malignant ovarian tissues. Brown (DAB) color shows Ikaros staining. (A) The image (original magnification $\mathrm{x} 400$ ) shows representative tissues that are normal, high differentiation carcinoma, moderate differentiation carcinoma and low differentiation carcinoma of ovarian cancer for Ikaros. (B) The image (x400) shows representative tissues that are FIGO stages I, II, III, and IV carcinoma of ovarian cancer for Ikaros.

$\beta$-tubulin (T4026, Sigma). All experiments were repeated at least three times.

RNA interference and transfection. All RNAi oligonucleotides were chemically synthesized by GenePharma Co. (Shanghai, China). These RNAi oligonucleotides were transfected into cells by using the Lipofectamine 2000 transfection kit (Invitrogen) according to the manufacturer's instructions. The siRNA sequences were as follows: nonspecific control (NC) 5'-UUCUCCGAACGUGUCACGU-3', Slug-1 siRNA human (S1) 5'-GGACUACCGCUGCUCCAUU-3', Slug-2 siRNA human (S2) 5'-GACCCACACAUUACCUUGU-3' and Slug-3 siRNA human (S3) 5'-GCACAAACAUGAGGAAUCU-3'.

Statistical analysis. The $\chi^{2}$ test was applied to test for a possible association between Ikaros expression and histological grade and type. Kruskal-Wallis test was performed to examine the significance of Ikaros expression among the groups as determined by IPP. Student's t-test was used to evaluate the difference between two different groups. All statistical analyses were performed using the SPSS software package (version 17.0, SPSS, Inc., Chicago, IL). Tests were 2 -sided and $\mathrm{P}<0.05$ was considered as statistically significant.

\section{Results}

Ikaros is expressed at high levels in human ovarian cancer tissues. To evaluate the expression levels of Ikaros in ovarian cancer, immunohistochemistry was performed on tissues from 87 cases of ovarian cancer and 10 cases of normal (only intact tissue samples were included). Compared with the weak positive staining of IK1 observed in $10 \%$ (1/10) of normal ovarian tissues, significant increase of positive staining of IK1 in $71 \%$ $(62 / 87)$ of ovarian cancer were observed (Table II, $\mathrm{P}<0.001)$.

We further analyzed the 10 normal ovarian tissues and 58 specimens of ovarian serous adenocarcinoma (OSA) tissues. The latter represents the most common subtype of epithelial ovarian cancer. Representative immunohistochemical findings of IK1 in tissue specimens are shown in Fig. 1. Significantly higher expression of IK1 was observed in malignant cancer tissues than 
Table II. Clinicopathological features of ovarian tissue with regard to the relative expression of Ikaros protein.

\begin{tabular}{lccc}
\hline Tissue & $\begin{array}{c}\text { No. of } \\
\text { specimens }\end{array}$ & $\begin{array}{c}\text { No. and ratio } \\
\text { of positive } \\
\text { expression }(\%)\end{array}$ \\
\hline Normal ovary & 10 & $1 / 10(10)$ \\
All malignant tumors & 87 & $62 / 87(71)^{\mathrm{a}}$ \\
Serous adenocarcinoma & 58 & $50 / 58(86)$ \\
Mucous adenocarcinoma & 6 & $2 / 6 \quad(33)$ \\
Adult granulosa cell tumor & 7 & $3 / 7 \quad(43)$ \\
Clear cell carcinoma & 5 & $2 / 5 \quad(40)$ \\
Dysgerminoma & 4 & $2 / 4 \quad(50)$ \\
Endometrioid adenocarcinoma & 3 & $2 / 3 \quad(67)$ \\
Sertoli-Leydig cell tumor & 1 & $0 / 1$ & $(0)$ \\
Mixed germ cell tumors & 1 & $0 / 1$ & $(0)$ \\
Malignant teratoma & 2 & $1 / 2 \quad(50)$ \\
\hline
\end{tabular}

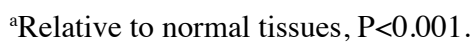

in normal ovarian tissues and significant differences were also observed among different pathology grades (Table III, $\mathrm{P}<0.001$ and Fig. 1A) and clinical stages of OSA (Table III, $\mathrm{P}=0.005$ ). Further analysis showed that there was a significant increase of IK1 expression in advanced-stage cancers (II, III, IV), where lymph nodes or distant metastases were present, in comparison to early-stage cancers (I) (Table III, $\mathrm{P}=0.001$ and Fig. 1B). These data indicated that IK1 may be involved in the metastasis of ovarian cancer cells.

Overexpression of IKI inhibits the proliferation of ovarian OSA cells. To investigate the potential role of IK1 in OSA, OSA cell line SKOV3 expressing low levels of IK1 were stably transfected with IK1 (SKOV3 ${ }^{\text {Flag-IK1 }}$ ) or the empty vector $\left(\mathrm{SKOV} 3^{\mathrm{EV}}\right)$. As shown in Fig. 2A, significant cell proliferation inhibition was observed in SKOV3 $3^{\text {Flag-IK1 }}$ cells compared with SKOV3 ${ }^{\mathrm{EV}}$ cells. In addition, IK1 overexpression elicited a significant increase in the number of SKOV3 cells in the $G_{0} / G_{1}$ phase with a concomi-
Table III. Correlation analysis of Ikaros and clinical manifestation of ovarian serous adenocarcinoma.

\begin{tabular}{lrcr}
\hline & & \multicolumn{2}{c}{ IOD } \\
\cline { 3 - 4 } Variables & $\mathrm{N}$ & Mean $\pm \mathrm{SD}$ & P-value \\
\hline Histology & & & $<0.001$ \\
Normal & 10 & $67901.3 \pm 38438.2$ & \\
$\mathrm{G}_{1}$ & 12 & $204987.6 \pm 71071.0$ & \\
$\mathrm{G}_{2}$ & 18 & $335607.7 \pm 100766.5$ & \\
$\mathrm{G}_{3}$ & 28 & $368952.4 \pm 92663.5$ & \\
& & & \\
Stage & & & \\
I & 26 & $270296.2 \pm 82500.65$ & \\
II & 13 & $341853.1 \pm 107934.8$ & \\
III & 14 & $408530.3 \pm 119316.5$ & \\
IV & 5 & $382052.8 \pm 63569.11$ & \\
Metastasis & & & \\
Negative (I) & 26 & $270296.2 \pm 82500.7$ & \\
Positive (II, III, IV) & 32 & $377305.6 \pm 109417.6$ & \\
\hline
\end{tabular}

tant decline in the $\mathrm{S}$ and $\mathrm{G}_{2} / \mathrm{M}$ phases (Fig. 2B). Consistent with the $\mathrm{G}_{1}$-arrest phenotype, $\mathrm{SKOV} 3^{\text {Flag-IK1 }}$ cells showed a dramatic elevation in the expression of the cell cycle inhibitors $(14,15)$, P21 and P27, along with a substantial decrease in the expression of the cell cycle inducers $(16,17)$, cyclin D1 and D2 (Fig. 2C). Similar results were observed in another OSA cell line HO8910 (data not shown), indicating that IK1-induced inhibition of cell proliferation is not SKOV3 cell specific.

Overexpression of IKI enhances migration and invasion of OSA cells. Since tissues analysis suggested that IK1 expression is associated with progression of ovarian cancer, we next investigated the effect of IK1 on the metastasis of ovarian cancer. The scratch wound healing assay (Fig. 3A), and transwell chamber assays (Fig. 3C) were performed to compare the migration and invasion capability between SKOV3 ${ }^{\mathrm{EV}}$ and SKOV3 $3^{\text {Flag-IK1 }}$ cells. At $24 \mathrm{~h}$, the SKOV3 $3^{\text {Flag-IK1 }}$ cells showed $\sim$-fold increase in migration and invasion capability (Fig. 3B
A

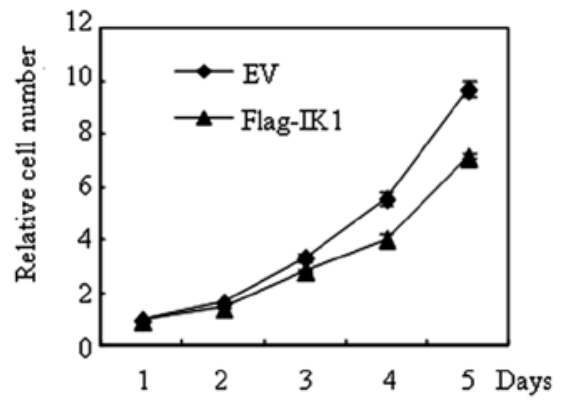

B

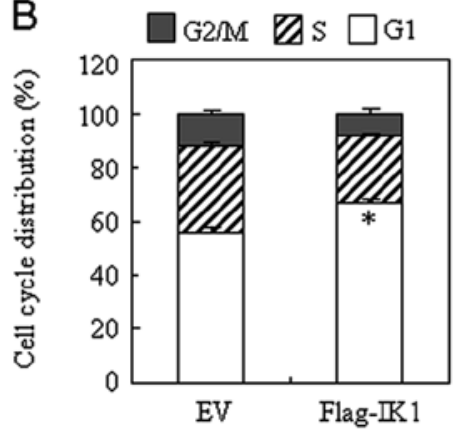

C

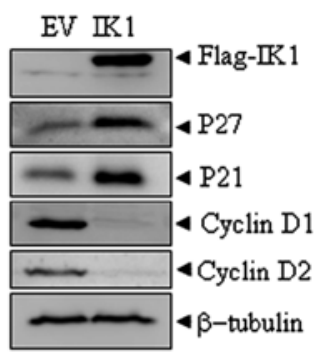

Figure 2. Overexpression of IK1 inhibits the proliferation of SKOV3 cells. (A) Cellular proliferation was markedly decreased in SKOV3 ${ }^{\text {Flag-IK1 }}$ cells compared with SKOV3 ${ }^{\mathrm{EV}}$ cells. (B) Cell cycle distribution of SKOV3 ${ }^{\mathrm{Flag}-\mathrm{IK} 1}$ and SKOV3 ${ }^{\mathrm{EV}}$ cells were determined by flow cytometry. (C) The indicated proteins were detected in $\mathrm{SKOV}^{\mathrm{EV}}$ or SKOV3 $3^{\text {Flag-IK1 }}$ cells by western blotting with $\beta$-tubulin as loading control. 
A

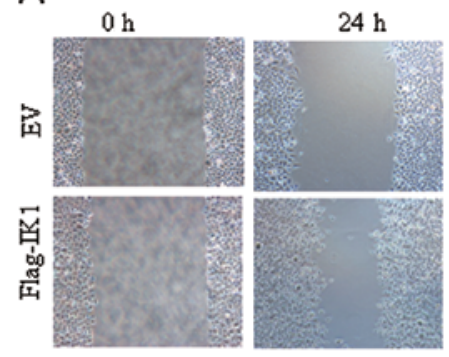

B

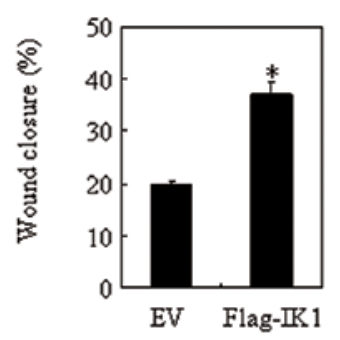

C

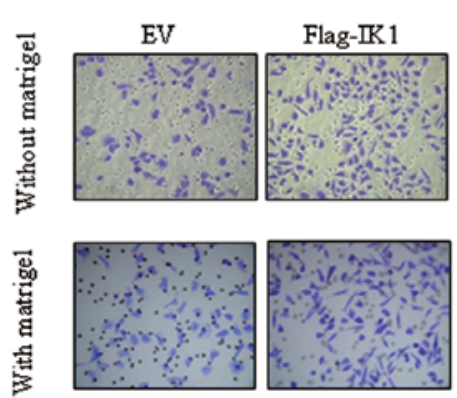

$\mathrm{F}$

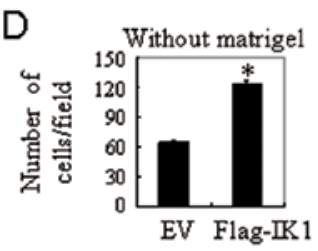

E
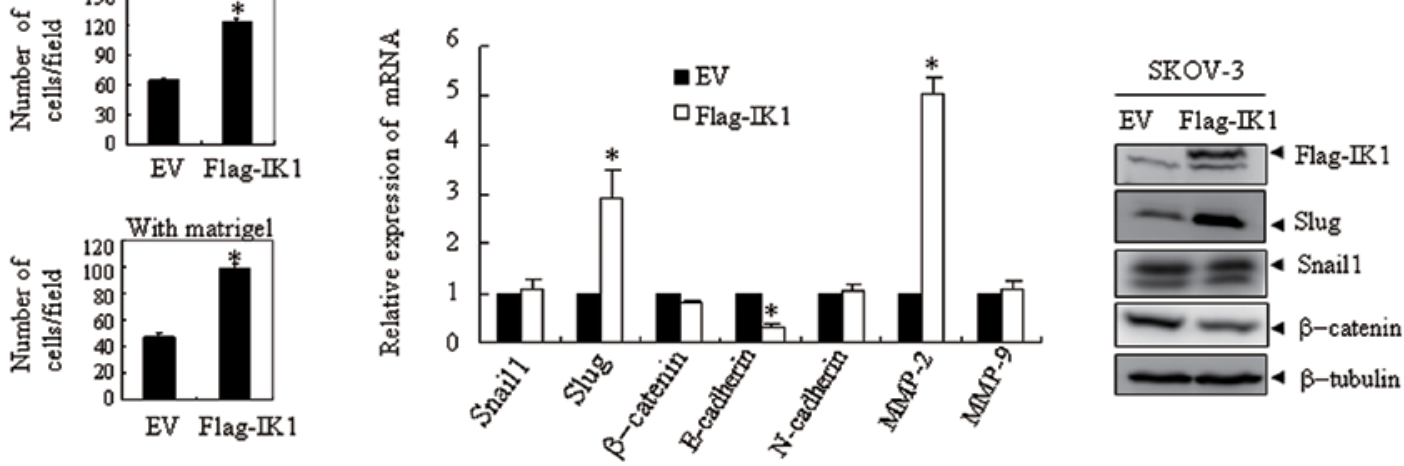

Figure 3. Overexpression of IK1 enhances migration and invasion of SKOV3 cells and influences expression of metastasis-related genes. SKOV3 $3^{\mathrm{Flag}-\mathrm{IK} 1}$ and SKOV3 ${ }^{\mathrm{EV}}$ cells were established as described in Materials and methods. Scratch-wound assay (A), transwell chamber migration (C, top panel), and matrigel invasion assay (C, bottom panel) were performed as illustrated in Materials and methods. The wound closure (\%) (B) and number of cells/field (D) were calculated. The indicated genes (E) were detected in SKOV3 ${ }^{\mathrm{EV}}$ or SKOV3 ${ }^{\mathrm{Flag}-\mathrm{IK} 1}$ cells by real-time quantitative PCR with GAPDH as internal control. The indicated proteins (F) were detected in $\mathrm{SKOV}^{\mathrm{EV}}$ or SKOV3 $3^{\text {Flag-IK1 }}$ cells by western blotting with $\beta$-tubulin as loading control. All values are expressed as the mean with bar SD of three independent experiments. ${ }^{\mathrm{P}}<0.01$ compared with SKOV3 ${ }^{\mathrm{EV}}$ cells.

A

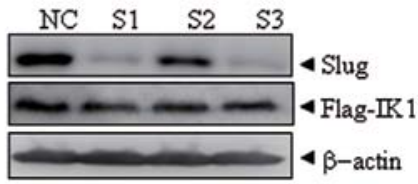

C

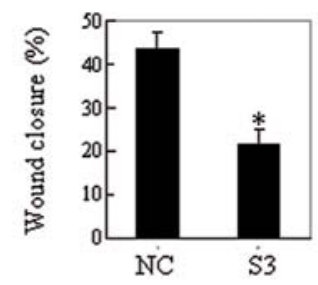

$\mathrm{D}$

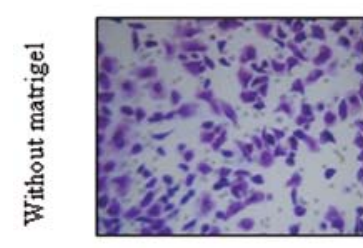

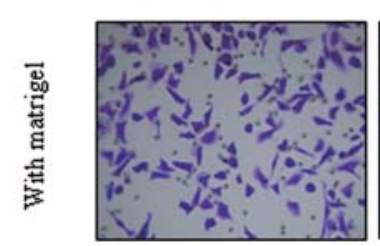

NC
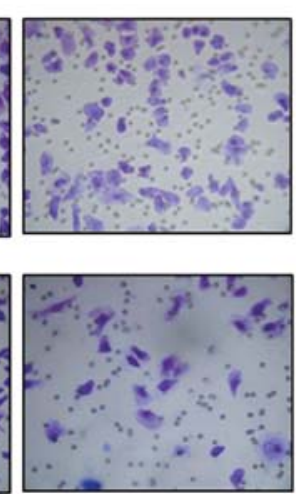

S3
B

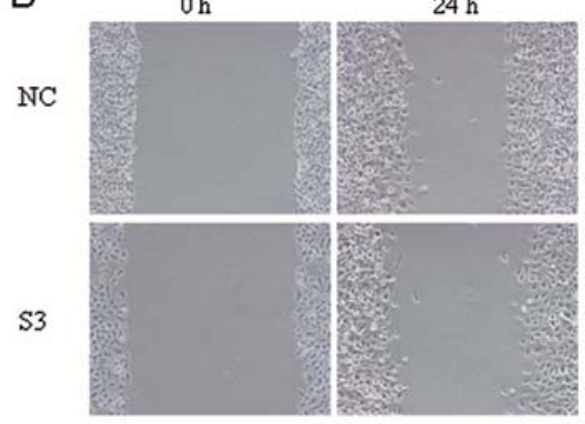

E
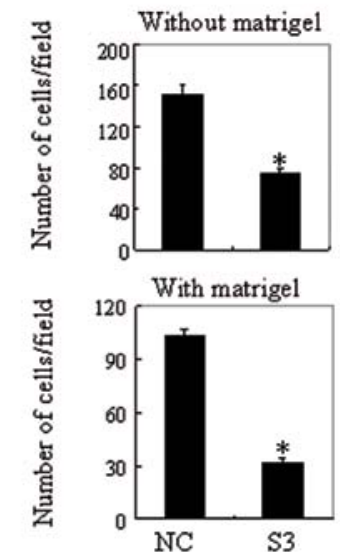

Figure 4. Knockdown of Slug significantly blocks IK1-induced migration and invasion in ovarian cancer cells. (A) SKOV3 ${ }^{\text {Flag-IK1 }}$ cells were transiently transfected with siRNA S1-3 against Slug or non-specific control vector (NC). Slug and Flag-IK1 proteins were blotted with $\beta$-actin as a loading control. Seventy-two hours after transfected with S3 or NC, the migration and invasion abilities of SKOV3 $3^{\text {Flag-IK1 }}$ cells were assessed by scratch-wound assay (B), transwell chamber migration (D, top panel) and matrigel invasion assay (D, bottom panel). The wound closure $(\%)(\mathrm{C})$ and number of cells/field $(\mathrm{E})$ were calculated and all values were expressed as the mean with bar SD. of three independent experiments. " $\mathrm{P}<0.01$ compared with NC transfected SKOV3 ${ }^{\text {Flag-IK1 }}$ cells. 
and D). Similar results were also observed in HO8910 cells (data not shown).

IK1 overexpression influences expression of metastasisrelated genes. To investigate how IK1 affects the metastatic process, a series of metastasis-related genes including Snail1, Slug, $\beta$-catenin, E-cadherin, N-cadherin, matrix metalloproteinase-2 (MMP-2), and matrix metalloproteinase-9 (MMP-9) were examined in SKOV3 $3^{\text {Flag-IK1 }}$ and SKOV3 ${ }^{\mathrm{EV}}$ cells. At mRNA level, overexpression of IK1 in SKOV3 cells resulted in a significant increase in the expression of Slug and MMP-2 and a significant decrease of E-cadherin. A slight downregulation of $\beta$-catenin was also observed (Fig. 3E). At the protein level, overexpression of IK1 in SKOV3 cells resulted in a marked increase of Slug and a decrease of $\beta$-catenin (Fig. 3F). Similar results were also observed in HO8910 cells (data not shown). These results indicated a potential role for Slug in IK1-induced migration and invasion of OSA cells.

Slug is responsible for IK1-induced migration and invasion of OSA cells. To illustrate whether Slug mediates IK1-induced migration and invasion of ovarian cancer, Slug was specifically knocked down by RNA interference. As shown in Fig. 4A, the target sequence S1 and S3 against Slug were efficient and specific, as the expression of Slug was efficiently suppressed with no effect on IK1 expression. Next, nonspecific control vector (NC) and S3 were transiently transfected into SKOV3 $3^{\text {Flag-IK1 }}$ cells. Seventy-two hours after transfection, the scratch wound healing assay (Fig. 4B), traswell migration assay, and matrigel invasion assay (Fig. 4D) were performed. The results revealed that the suppression of Slug expression could significantly block IK1-induced migration and invasion of SKOV3 ${ }^{\text {Flag-IK1 }}$ cells (Fig. 4C and E). These results indicated that Slug contributed to IK1-induced cell migration and invasion in ovarian cancer cells.

\section{Discussion}

The ability to metastasize makes ovarian cancer a fatal disease and a significant number of patients treated for the localized disease ultimately present with metastases. A better understanding of molecular events that contribute to tumor invasion and metastasis is crucial for developing novel treatment strategies for ovarian cancer.

Although most studies on Ikaros are restricted to the hematopoietic system, Ikaros plays a role in other tissues (9,18-21). In this study, we show for the first time that Ikaros is involved in the metastasis and invasion of ovarian cancer cells. First, higher expression of IK1 was observed in malignant ovarian cancer tissues and was significantly associated with the high FIGO stage and low differentiation state in OSA; second, overexpression of IK1 in OSA cells resulted in enhanced cell invasion and metastasis. Consistent with our results, Yamamoto et al (19) reported that IK1 is involved in migration and invasion of extravillous trophoblasts in early placentation, although the underlying mechanism is unknown.

Slug is a member of the Snail family of zinc finger transcription factors that play a central role in the patterning of vertebrate (22). Recent evidence showed that Slug is upregulated in metastatic breast cancer, mesothelioma, and ovarian cancer, and plays an important role in cancer invasion $(23,24)$. It is interesting to note that Slug is also implicated in IK1-induced cell migration and invasion. While IK1 significantly upregulated the expression of Slug, knocking down Slug abrogated IK1-induced cell migration and invasion. It is known that Slug could regulate the cell metastasis and invasion through regulation of the expression of junctional proteins such as E-cadherin and matrix metalloproteinase, which can degrade the ECM components and is believed to play a major role in invasion and metastasis (25-29). In support of this, E-cadherin and MMP-2, two Slug target genes, could also be downregulated and upregulated, respectively. Of note, the effect of IK1 on Slug is relatively specific, as Snail1, another member of the Snail family that plays a functional role in ovarian cancer metastasis, was not altered by IK1 overexpression.

One intriguing finding of this study is that IK1 plays a dual role in ovarian cancer cells, inhibiting cell proliferation on one hand and increasing metastatic ability on the other hand. Although apparently conflicting, this kind of phenomenon is not restricted to the Ikaros family. TGF- $\beta$ signaling has been shown to function as a double-edged sword in ovarian cancer development, a tumor suppressor in early tumorigenesis but a tumor enhancer in advanced-stage cancer $(30,31)$. Similar situation may exist for IK1 in the progression of ovarian cancer. In our microarrays study, we found metastatic, poorly differentiated cancer tissues tend to express higher levels of IK1 protein, indicative of the contribution of IK1 in ovarian cancer progression.

In conclusion, we provide evidence that overexpression of IK1 in ovarian cancer cells play a dual role in proliferation, migration and invasion and Slug upregulation contributes to IK1-induced increase of cell migration and invasion. This study reveals a new link between a hematopoietic transcription factor and the metastasis of ovarian cancer cells. Further studies on Ikaros-induced Slug upregulation may provide novel targets for inhibiting ovarian cancer metastasis.

\section{Acknowledgements}

This study was supported in part by grants from National Basic Research Program of China(973 Program) (No. 2009CB918404, 2010CB912104), National Natural Science Foundation of China (31100980, 81172322, 81070433, 91013008), Science and Technology Committee of Shanghai (11JC1406500), Innovation Program of Shanghai Municipal Education Commission (13YZ028) and SMC Program of Shanghai Jiao Tong University.

\section{References}

1. Jemal A, Siegel R, Xu J and Ward E: Cancer statistics, 2010. CA Cancer J Clin 60: 277-300, 2010.

2. Naora $\mathrm{H}$ and Montell DJ: Ovarian cancer metastasis: integrating insights from disparate model organisms. Nat Rev 5: 355-366, 2005.

3. Molnar A and Georgopoulos K: The Ikaros gene encodes a family of functionally diverse zinc finger DNA-binding proteins. Mol Cell Biol 14: 8292-8303, 1994.

4. Koipally J and Georgopoulos K: A molecular dissection of the repression circuitry of Ikaros. J Biol Chem 277: 27697-27705, 2002.

5. Georgopoulos K, Bigby M, Wang JH, et al: The Ikaros gene is required for the development of all lymphoid lineages. Cell 79: 143-156, 1994.

6. Dumortier A, Kirstetter P, Kastner P and Chan S: Ikaros regulates neutrophil differentiation. Blood 101: 2219-2226, 2003. 
7. Wang JH, Nichogiannopoulou A, Wu L, et al: Selective defects in the development of the fetal and adult lymphoid system in mice with an Ikaros null mutation. Immunity 5: 537-549, 1996

8. Payne KJ, Huang G, Sahakian E, et al: Ikaros isoform $\mathrm{x}$ is selectively expressed in myeloid differentiation. J Immunol 170 3091-3098, 2003.

9. Ezzat S, Mader R, Yu S, Ning T, Poussier P and Asa SL: Ikaros integrates endocrine and immune system development. J Clin Invest 115: 1021-1029, 2005.

10. Yang L, Luo Y and Wei J: Integrative genomic analyses on Ikaros and its expression related to solid cancer prognosis. Oncol Rep 24: 571-577, 2010.

11. He LC, Xu HZ, Gu ZM, et al: Ikaros is degraded by proteasomedependent mechanism in the early phase of apoptosis induction. Biochem Biophys Res Commun 406: 430-434, 2011.

12. Zhao KW, Li X, Zhao Q, et al: Protein kinase Cdelta mediates retinoic acid and phorbol myristate acetate-induced phospholipid scramblase 1 gene expression: its role in leukemic cell differentiation. Blood 104: 3731-3738, 2004.

13. Xavier LL, Viola GG, Ferraz AC, et al: A simple and fast densitometric method for the analysis of tyrosine hydroxylase immunoreactivity in the substantia nigra pars compacta and in the ventral tegmental area. Brain Res Brain Res Protoc 16: 58-64, 2005.

14. Sherr CJ and Roberts JM: CDK inhibitors: positive and negative regulators of G1-phase progression. Genes Dev 13: 1501-1512, 1999.

15. Alt JR, Gladden AB and Diehl JA: p21(Cip1) promotes cyclin D1 nuclear accumulation via direct inhibition of nuclear export. J Biol Chem 277: 8517-8523, 2002.

16. Masamha CP and Benbrook DM: Cyclin D1 degradation is sufficient to induce G1 cell cycle arrest despite constitutive expression of cyclin E2 in ovarian cancer cells. Cancer Res 69: 6565-6572, 2009.

17. Ely S, Di Liberto M, Niesvizky R, et al: Mutually exclusive cyclin-dependent kinase 4/cyclin D1 and cyclin-dependent kinase 6/cyclin D2 pairing inactivates retinoblastoma protein and promotes cell cycle dysregulation in multiple myeloma. Cancer Res 65: 11345-11353, 2005.

18. Ezzat S and Asa SL: The emerging role of the Ikaros stem cell factor in the neuroendocrine system. J Mol Endocrinol 41: 45-51, 2008
19. Yamamoto E, Ito T, Abe A, et al: Ikaros is expressed in human extravillous trophoblasts and involved in their migration and invasion. Mol Hum Reprod 11: 825-831, 2005.

20. Ezzat S, Yu S and Asa SL: Ikaros isoforms in human pituitary tumors: distinct localization, histone acetylation, and activation of the 5' fibroblast growth factor receptor-4 promoter. Am J Pathol 163: 1177-1184, 2003.

21. Elliott J, Jolicoeur C, Ramamurthy V and Cayouette M: Ikaros confers early temporal competence to mouse retinal progenitor cells. Neuron 60: 26-39, 2008.

22. Alves CC, Carneiro F, Hoefler H and Becker KF: Role of the epithelial-mesenchymal transition regulator Slug in primary human cancers. Front Biosci 14: 3035-3050, 2009.

23. Kurrey NK, K A and Bapat SA: Snail and Slug are major determinants of ovarian cancer invasiveness at the transcription level. Gynecol Oncol 97: 155-165, 2005.

24. Catalano A, Rodilossi S, Rippo MR, Caprari P and Procopio A: Induction of stem cell factor/c-Kit/slug signal transduction in multidrug-resistant malignant mesothelioma cells. J Biol Chem 279: 46706-46714, 2004.

25. Liotta LA, Abe S, Robey PG and Martin GR: Preferential digestion of basement membrane collagen by an enzyme derived from a metastatic murine tumor. Proc Natl Acad Sci USA 76: 2268-2272, 1979.

26. Batlle E, Sancho E, Franci C, et al: The transcription factor snail is a repressor of E-cadherin gene expression in epithelial tumour cells. Nat Cell Biol 2: 84-89, 2000.

27. Nieto MA: The snail superfamily of zinc-finger transcription factors. Nat Rev Mol Cell Biol 3: 155-166, 2002.

28. Shih JY and Yang PC: The EMT regulator slug and lung carcinogenesis. Carcinogenesis 32: 1299-1304, 2011.

29. Kenny HA and Lengyel E: MMP-2 functions as an early response protein in ovarian cancer metastasis. Cell cycle 8: 683-688, 2009

30. Akhurst RJ and Derynck R: TGF-beta signaling in cancer - a double-edged sword. Trends Cell Biol 11: S44-S51, 2001.

31. Chou JL, Chen LY, Lai HC and Chan MW: TGF-beta: friend or foe? The role of TGF-beta/SMAD signaling in epigenetic silencing of ovarian cancer and its implication in epigenetic therapy. Expert Opin Ther Targets 14: 1213-1223, 2010. 